PROCEEDINGS OF THE

AMERICAN MATHEMATICAL SOCIETY

Volume 132, Number 7, Pages 1873-1884

S 0002-9939(03)07287-3

Article electronically published on November 26, 2003

\title{
SUBGROUP SEPARABILITY OF GRAPHS OF ABELIAN GROUPS
}

\author{
V. METAFTSIS AND E. RAPTIS \\ (Communicated by Stephen D. Smith)
}

\begin{abstract}
In the present paper we give necessary and sufficient conditions for the subgroup separability of the fundamental group of a finite graph of groups with finitely generated abelian vertex groups.
\end{abstract}

\section{INTRODUCTION}

A group $G$ is called subgroup separable or locally extended residually finite (LERF) if every finitely generated subgroup of $G$ is closed in the profinite topology, the topology whose open basis consists of the cosets of finite index subgroups of $G$.

There are other equivalent definitions of subgroup separability. The most commonly used is that $G$ is subgroup separable if for every finitely generated subgroup $H$ of $G, H$ is the intersection of the finite index subgroups of $G$ containing $H$. Or that for every finitely generated subgroup $H$ of $G$ and every $g \notin H$ there is a normal subgroup $N$ of finite index in $G$ such that $g \notin N H$. This is equivalent to saying that $g N \notin H N / N$ or that $\bigcap_{N \in \mathcal{N}} N H=H$ where $\mathcal{N}$ is the set of all normal subgroups of finite index in $G$.

Historically, subgroup separability was first shown to be a property of free groups by M. Hall, Jr. in [10]. The geometric motivation for studying subgroup separability of groups was given in [16] and [17. More specifically, P. Scott proved that subgroup separability allows certain immersions to lift to an embedding in a finite cover. This result is of particular importance in 3-manifold topology. Since then many people have worked on the subject. For the families of groups that are known to satisfy subgroup separability the reader should consult the papers of Gitik [8] and Wise [19] and the references cited there.

On the other hand, there are very few known examples of finitely presented, residually finite, non-subgroup separable groups. The first example in the literature is the group $F_{2} \times F_{2}$, shown by Mihailova in 13 to have an unsolvable generalised word problem, thus being non-subgroup separable.

The second example in the literature is the family $B N$ of all groups with an HNN presentation of the form $\left\langle t, K \mid t K t^{-1}=A\right\rangle$ where $A \subsetneq K$. Such an HNNextension is called ascending. The above groups are not subgroup separable by the

Received by the editors February 18, 2002 and, in revised form, March 7, 2003.

2000 Mathematics Subject Classification. Primary 20E05, 20E06, 20 E26.

Key words and phrases. Subgroup separable groups (LERF), residually finite groups, HNNextensions, graphs of groups. 
results of Blass and Neumann in [5. Notice that the $B N$ groups include the residually finite, non-subgroup separable Baumslag-Solitar groups, namely the groups $B S_{n, m}=\left\langle x, a \mid x a^{n} x^{-1}=a^{m}\right\rangle$ with either $|n|=1$ or $|m|=1$ and $|m| \neq|n|$. Shalen recently showed [18] that non-subgroup separable Baumslag-Solitar groups cannot be subgroups of 3-manifold groups.

The third example in the literature (the first non-subgroup separable 3-manifold group) was discovered by Burns, Karrass and Solitar in [6] and is the group

$$
B K S=\left\langle x, \alpha, \beta \mid x \alpha x^{-1}=\alpha, x \beta x^{-1}=\alpha \beta\right\rangle .
$$

Furthermore, it was shown by Long and Niblo 11] and subsequently by Niblo and Wise [14 that the group $B K S$ contains a subgroup of index two that is isomorphic to a subgroup of the group

$$
\begin{gathered}
L=\langle x, y, \alpha, \beta \mid[x, \alpha]=[y, \beta]=[\alpha, \beta]=1\rangle \\
\cong\langle\alpha, x \mid[\alpha, x]=1\rangle \underset{\alpha}{*}\langle\alpha, \beta \mid[\alpha, \beta]=1\rangle \underset{\beta}{*}\langle\beta, y \mid[\beta, y]=1\rangle .
\end{gathered}
$$

The above group $L$ is a right-angled Artin group. For more details, terminology and a recent result on right-angled Artin groups the reader should consult the work of Bestvina and Brady in [4] and the references cited there. Niblo and Wise in [14] show that the fundamental group of a graph manifold is subgroup separable if and only if it does not contain a subgroup isomorphic to $L$. The group $L$ seems to be the key point for the results of the present paper also. In fact, summarising the results of the present work together with the results of [12], we have shown the following.

Theorem. The fundamental group of a finite graph of groups with finitely generated abelian vertex groups is subgroup separable if and only if it does not contain a subgroup isomorphic to any of the following:

(1) $L$,

(2) $B K S$,

(3) an ascending $H N N$-extension with finitely generated abelian base group,

(4) a non-residually finite $H N N$-extension with finitely generated abelian base group.

This last category of groups was completely characterised in [3].

Furthermore, the non-subgroup separable example of Mihailova in [13] contains a subgroup isomorphic to $L$ (see Corollary 1), and the examples of Gitik and Rips in [9] and of Allenby and Doniz in 1] are special cases of our main theorem (see Corollaries 3 and 2).

These give rise to the following interesting question.

Question. Can we construct a finitely presented, residually finite, non-subgroup separable group that contains neither a finite index subgroup of BKS nor a finite index subgroup of any member of the family BN of groups?

The present paper is structured as follows.

In Section 2 we give three lemmas which we need in the subsequent results.

In Section 3 we give necessary and sufficient conditions for the subgroup separability of groups that can be realised as fundamental groups of graphs of groups with finitely generated abelian vertex and edge groups. 
In Section 4 we give some applications of our Theorem 1. In fact, we show that the non-subgroup separable example of [13] contains a subgroup isomorphic to $L$ and also that the two examples of [9] and [1] are special cases of our main theorem.

\section{Some InTERMEDiate RESUlts}

In the sequel we shall make frequent use of the following lemma of Scott in [16].

Lemma 1 (16]). If $G$ is a subgroup separable group, then any subgroup of $G$ is subgroup separable and so is any group $K$ that contains $G$ as a subgroup of finite index.

The following lemma is probably known, but we decided to include its proof since we could not find a reference for it.

Lemma 2. Let $K$ be a group and let $G$ be the $H N N$-extension

$$
G=\left\langle t, K \mid t A t^{-1}=B\right\rangle
$$

such that the associated subgroups $A$ and $B$ are finite. Then $G$ is subgroup separable if and only if $K$ is subgroup separable.

Proof. If $G$ is subgroup separable, then the result follows from Lemma 1 .

Conversely, assume that $K$ is subgroup separable. Then $K$ is residually finite, and we can easily show that $G$ is also residually finite (see, for example, exercise 26 , page 47 in [7]). Hence, for every $\alpha \in A$ we can find a finite index normal subgroup of $G$, say $N_{\alpha}$, such that $\alpha \notin N_{\alpha}$. Similarly, for every $\beta \in B$ we can always find a finite index normal subgroup of $G$, say $N_{\beta}$, such that $\beta \notin N_{\beta}$. Hence, the group $N=\left(\bigcap_{\alpha \in A} N_{\alpha}\right) \cap\left(\bigcap_{\beta \in B} N_{\beta}\right)$ is a normal subgroup of finite index in $G$ that meets $A$ and $B$ trivially.

Now let $(\mathcal{G}, X)$ be a graph of groups with a single vertex $v$ with vertex group $K$ and a single edge $e$ (loop) with edge group $A$ such that $\pi_{1}(\mathcal{G}, X)=G$. From BassSerre theory (see [7]) we know that the subgroup $N$ is the fundamental group of a graph of groups with vertex groups $N \cap g K g^{-1}$ where $g$ runs over a suitable finite set of $(N, K)$ double coset representatives and edge groups $N \cap h A h^{-1}$ where $h$ runs over a suitable finite set of $(N, A)$ double coset representatives. Since $K$ is subgroup separable, so is $g K g^{-1}$, and hence $N \cap g K g^{-1}$ is also subgroup separable. Since $N$ is normal in $G$, and $N \cap A$ is trivial, it follows that $N \cap h A h^{-1}=\{1\}$, for any element $h \in G$. Hence, $N$ is the free product of a finite number of subgroup separable groups and (possibly) a free group. But such a group is known to be subgroup separable. Furthermore, $G$ is a finite extension of $N$; hence $G$ is subgroup separable.

The following lemma is the second part of the proof of Lemma 2 in [12].

Lemma 3 (12). Let $G$ be a finitely generated group and $H$ a finitely generated, abelian, normal subgroup of $G$. If $G / H^{n}$ is subgroup separable for every $n \in \mathbb{N}$, then $G$ is subgroup separable.

Let us introduce some notation. Assume that $X$ is a graph. On $X$ we define as usual (see, for example, [7]) $V X$ and $E X$, the sets of vertices and edges of $X$ respectively, together with three functions $\iota: E X \rightarrow V X, \tau: E X \rightarrow V X$ and ${ }^{-}: E X \rightarrow V X$ such that for all $e \in E X, e \neq \bar{e}, e=\overline{\bar{e}}$ and $\tau(e)=\iota(\bar{e})$.

Suppose now that $G$ is the fundamental group of a graph $T$ of groups. Then, by Bass-Serre theory (see [7]), we know that there is a tree $\Gamma$ on which $G$ acts such 
that the quotient $G \backslash \Gamma$ equals the underlying graph $T$, and the group associated to a vertex $w$ of $T$ is the stabiliser of a vertex $v$ of $\Gamma$ that projects to $w$. Also, a change in the choice of $v$ alters this group by conjugacy. So, in the sequel, we shall always choose a maximal subtree $T_{0}$ of $T$ and a subtree $\Gamma_{0}$ of $\Gamma$ whose projection to $T$ induces a bijection from $\Gamma_{0}$ to $T_{0}$. This choice of $\Gamma_{0}$ gives a choice for a given vertex and edge group of $T_{0}$. Also, when necessary, we shall make choices of vertices and edges in $\Gamma \backslash \Gamma_{0}$ in order to extend $\Gamma_{0}$ to a subtree of $\Gamma$, say $\bar{\Gamma}$, such that there is a bijection between the edges of $\bar{\Gamma}$ and the edges of $T$. We will denote the stabiliser of $f$ in $\Gamma_{0}$ by $G_{f}$. Assuming that the edge $e$ projects in $T_{0}$ to $e$, we shall identify $G_{f}$ with the edge group of $f$. Similarly, if a vertex $w$ of $\Gamma_{0}$ projects to $v$ in $T_{0}$, we shall identify the stabiliser of $w$ in $\Gamma, G_{w}$, with the vertex group of $v$.

Now let $T$ be a tree with three vertices $v_{i}, i=1,2,3$ and two geometric edges $e_{i}, i=1,2$ such that $v_{1}$ and $v_{3}$ are the extremal vertices of $T$. Let $(\mathcal{G}, T)$ be the graph of groups with underlying graph $T$ and finitely generated abelian vertex and edge groups. We denote by $G_{v_{i}}$ the corresponding vertex groups and by $G_{e_{i}}$ the corresponding edge groups. Since $T$ is a tree, we have that $G_{e_{i}}=G_{\bar{e}_{i}}$ for every $e_{i}$, $i=1,2$. From the action of $G$ on the tree $\Gamma$, it is automatic that $G_{e_{i}} \leq G_{\iota\left(e_{i}\right)}$ and that $G_{e_{i}} \leq G_{\tau\left(e_{i}\right)}, i=1,2$.

Lemma 4. Let $(\mathcal{G}, T)$ be as above and assume that $e_{1}, e_{2} \in E T$ such that $e_{1} \neq \overline{e_{2}}$. Suppose also that all edge groups are proper subgroups of the vertex groups that these edges connect. Then the following statements are equivalent:

(1) $G=\pi_{1}(\mathcal{G}, T)$ is subgroup separable;

(2) $G_{e_{1}} \cap G_{e_{2}}$ is of finite index in $G_{e_{1}}$ or in $G_{e_{2}}$;

(3) $G$ does not contain a subgroup isomorphic to $L$.

Proof. $1 \Rightarrow 2$. From its definition, and standard Bass-Serre theory ([7]), $G=$ $\pi_{1}(\mathcal{G}, T)$ is the free product with amalgamation of the form

$$
\pi_{1}(\mathcal{G}, T)=G_{v_{1}} \underset{G_{e_{1}}}{*} G_{v_{2}} \underset{G_{e_{2}}}{*} G_{v_{3}}
$$

where all groups are finitely generated abelian groups. Hence, all vertex and edge groups are subgroup separable.

Let $G$ be subgroup separable, and assume that neither $G_{e_{1}}$ nor $G_{e_{2}}$ is finite. If $\left|G_{e_{1}}: G_{e_{1}} \bigcap G_{e_{2}}\right|=\infty=\left|G_{e_{2}}: G_{e_{1}} \bigcap G_{e_{2}}\right|$, then neither $\left|G_{v_{2}}: G_{e_{1}}\right|<\infty$ nor $\mid G_{v_{2}}$ : $G_{e_{2}} \mid<\infty$. Indeed, if for example $\left|G_{v_{2}}: G_{e_{1}}\right|<\infty$, then $\left|G_{e_{2}}: G_{e_{1}} \cap G_{e_{2}}\right|<\infty$, a contradiction.

From the discussion before Lemma 4 we can choose $\Gamma_{0}$ to be a subtree of $\Gamma$ whose projection to $T$ induces a bijection from $\Gamma_{0}$ to $T$. Let $w_{i}$ denote the vertex of $\Gamma_{0}$ that projects to $v_{i}$, and $f_{i}$ the edge of $\Gamma_{0}$ that projects to $e_{i}$, such that $G_{w_{i}}=G_{v_{i}}$, $i=1,2,3$ and $G_{f_{i}}=G_{e_{i}}, i=1,2$. Let $H$ denote the subgroup of $G$ generated by $G_{w_{2}}, a G_{w_{2}} a^{-1}$ and $b G_{w_{2}} b^{-1}$ where $a$ and $b$ are coset representatives of $G_{w_{2}}$ in $G$ with $a \in G_{w_{1}} \backslash G_{f_{1}}$ and $b \in G_{w_{3}} \backslash G_{f_{2}}$.

Then $a G_{w_{2}} a^{-1}$ is the stabiliser of $a w_{2}$ that is different from $w_{2}$ by the choice of $a$. Similarly, $b G_{w_{2}} b^{-1}$ is the stabiliser of $b w_{2}$ that is different from $w_{2}$ and $a w_{2}$, by the choice of $b$. The subgraph of $\Gamma$ with vertices $a w_{2}, w_{2}$ and $b w_{2}$ is a tree with extremal vertices $a w_{2}$ and $b w_{2}$, and so $H$ is the fundamental group of a graph of groups with graph as the above tree. Hence, $H$ is of the form

$$
a G_{w_{2}} a^{-1} \underset{H_{1}}{*} G_{w_{2}} \underset{H_{2}}{*} b G_{w_{2}} b^{-1} .
$$


The amalgamating subgroup $H_{1}$ is the intersection $a G_{w_{2}} a^{-1} \cap G_{w_{2}}$ and so is the stabiliser of the segment joining $w_{2}$ and $a w_{2}$ in $\Gamma$. Since $a$ stabilises $w_{1}$, and $w_{1}$ and $w_{2}$ are adjacent, this segment consists of the two edges $f_{1}$ and $a f_{1}$. Thus, $a G_{w_{2}} a^{-1} \cap G_{w_{2}}=G_{f_{1}} \cap a G_{f_{1}} a^{-1}$. Since $G_{f_{1}}$ and $a$ lie in the abelian group $G_{w_{1}}$ it follows that $G_{f_{1}}=a G_{f_{1}} a^{-1}$; so $H_{1}=G_{f_{1}}$. Similarly, $H_{2}=G_{f_{2}}$. Since $G_{w_{2}}$ contains $G_{f_{1}}$ and $G_{f_{2}}$ with infinite index, we can choose $x \in a G_{w_{2}} a^{-1}$ of infinite order such that $\langle x\rangle \cap G_{f_{1}}=\{1\}$ and $y \in b G_{w_{2}} b^{-1}$ such that $\langle y\rangle \cap G_{f_{2}}=\{1\}$. This implies that $\langle x\rangle \cap\langle y\rangle=\{1\}$. Also, since $\left|G_{f_{1}}: G_{f_{1}} \cap G_{f_{2}}\right|=\infty$ we can choose $\beta_{1} \in G_{f_{1}}$ such that $\beta_{1}$ has infinite order and $\left\langle\beta_{1}\right\rangle \cap G_{w_{2}}=\{1\}$. Similarly, since $\left|G_{f_{2}}: G_{f_{1}} \cap G_{f_{2}}\right|=\infty$, we can choose $\beta_{2} \in G_{f_{2}}$ of infinite order with $\left\langle\beta_{1}\right\rangle \cap\left\langle\beta_{2}\right\rangle=\{1\}$. Let $L$ be the subgroup of $G$ generated by $\left\langle x, y, \beta_{1}, \beta_{2}\right\rangle$. From their choices we have that $x, \beta_{1} \in a G_{w_{2}} a^{-1}$, $\beta_{1}, \beta_{2} \in G_{w_{2}}$ and $\beta_{2}, y \in b G_{w_{2}} b^{-1}$. So, due to the form of $H$ and the choices of $x, y, \beta_{1}, \beta_{2}, L$ has a presentation

$$
L=\left\langle x, \beta_{1} \mid\left[x, \beta_{1}\right]=1\right\rangle \underset{\beta_{1}}{*}\left\langle\beta_{1}, \beta_{2} \mid\left[\beta_{1}, \beta_{2}\right]=1\right\rangle \underset{\beta_{2}}{*}\left\langle\beta_{2}, y \mid\left[\beta_{2}, y\right]=1\right\rangle .
$$

But $L$ was shown in [11] to be a non-subgroup separable group (containing a finite index subgroup of $B K S$ ), a contradiction to our assumption that every subgroup of $G$ is subgroup separable by Lemma 1

$2 \Rightarrow 1$. Suppose that $G_{e_{1}}$ is finite. The group $M=G_{v_{2}}{ }_{G_{e_{2}}} G_{v_{3}}$ is a subgroup of $G$. Since $G_{v_{2}}$ and $G_{v_{3}}$ are abelian, the group $G_{e_{2}}^{n}$ is normal in $G_{v_{2}}$ and $G_{v_{3}}$ and hence in $M$ for every integer $n \geq 1$. Also, $M / G_{e_{2}}^{n}$ is a free product of abelian groups with finite amalgamation, hence by the results of Allenby and Gregorac in [2], is subgroup separable for every $n \in \mathbb{N}$. Therefore, by Lemma $3 \quad M$ is subgroup separable and so is $G$ since it is a free product of subgroup separable groups with finite amalgamation, again by the results in [2]. A similar argument works if $G_{e_{2}}$ is finite.

Finally, if neither $G_{e_{1}}$ nor $G_{e_{2}}$ is finite, assume that $\left|G_{e_{1}}: G_{e_{1}} \cap G_{e_{2}}\right|<\infty$. Let $H=G_{e_{1}} \cap G_{e_{2}}$. Then $H^{n}$ is normal in each vertex group of $(\mathcal{G}, T)$ and so is normal in $G$ for every $n \in \mathbb{N}$. Hence,

$$
G / H^{n}=G_{v_{1}} / H^{n} \underset{G_{e_{1}} / H^{n}}{*} G_{v_{2}} / H^{n} \underset{G_{e_{2}} / H^{n}}{*} G_{v_{3}} / H^{n} .
$$

Obviously, $\left|G_{e_{1}}: H^{n}\right|<\infty$. Moreover, $G_{v_{2}} / H_{G_{e_{2}} / H^{n}}^{*} G_{v_{3}} / H^{n}$ is subgroup separable and so $G / H^{n}$ is subgroup separable. Then, by Lemma 3, $G$ is subgroup separable.

$1 \Rightarrow 3$. Since $G$ is subgroup separable, then it cannot contain a subgroup isomorphic to $L$ by Lemma 1 .

$3 \Rightarrow 2$. In the proof that $1 \Rightarrow 2$, we showed that if (2) does not hold, then $G$ contains a subgroup isomorphic to $L$. Thus, if $G$ does not contain such a subgroup, it follows that (2) holds.

\section{GRaphs of ABELian gRoups}

Let us assume now that $(\mathcal{G}, T)$ is a graph of groups with $T$ a finite tree and such that every vertex and edge group of $T$ is a finitely generated abelian group. Furthermore, assume that in $(\mathcal{G}, T)$, no edge group is equal to either of the vertex groups that this edge connects. Having in mind the discussion before Lemma 4, we can show the following. 
Lemma 5. Let $(\mathcal{G}, T)$ be as above. Then the following statements are equivalent:

(1) $G=\pi_{1}(\mathcal{G}, T)$ is subgroup separable;

(2) for every connected subgraph $Y$ of $T$, the group $\bigcap_{e \in E Y} G_{e}$ has finite index in $G_{e}$ for at least one $e \in E Y$;

(3) $G$ does not contain a subgroup isomorphic to $L$.

Proof. $1 \Rightarrow 2$. Let $\Gamma$ be the $G$-tree and $\Gamma_{0}$ be the subtree of $\Gamma$ whose projection to $Y$ induces a bijection. Based on the discussion before Lemma 4, we shall identify the vertices and edges of $\Gamma_{0}$ to those of $T$.

Let us assume that $G$ is subgroup separable. We shall show that for every connected subgraph $Y$ of $T$, the group $\bigcap_{e \in E Y} G_{e}$ has finite index in at least one $G_{e}, e \in E Y$. The proof will be by induction on the number of geometric edges that $Y$ contains.

If $Y$ has one geometric edge, the result is trivial, and if $Y$ has two geometric edges, the result follows from Lemma 4. Now let $m>2$ be the number of the geometric edges of $Y$ and let $e_{1}, \ldots, e_{m}$ be a collection of edges of $E Y$ such that $e_{i} \neq \overline{e_{j}}$ for every $i, j \in\{1, \ldots, m\}$. For simplicity, assume also that $e_{1}$ and $e_{m}$ are edges that connect extremal vertices of $Y$ to the rest of the tree. From the induction hypothesis, we have that $\bigcap_{i=1}^{m-1} G_{e_{i}}$ has finite index in $G_{e_{r}}$ for some $r$ with $1 \leq r \leq m-1$. Similarly, $\bigcap_{i=2}^{m} G_{e_{i}}$ has finite index in $G_{e_{s}}$ for some $s$ with $2 \leq s \leq m$. If $s=r$, then $\bigcap_{i=1}^{m} G_{e_{i}}$ has finite index in $G_{e_{s}}$ and the result is proved. If $s \neq r$, then assume that $\bigcap_{i=1}^{m} G_{e_{i}}$ has infinite index in every $G_{e_{i}}, 1 \leq i \leq m$.

Since $\bigcap_{i=1}^{m} G_{e_{i}}$ equals the intersection of $\bigcap_{i=2}^{m} G_{e_{i}}$ and $\bigcap_{i=1}^{m-1} G_{e_{i}}$, it follows that $\bigcap_{i=1}^{m} G_{e_{i}}$ has finite index in $G_{e_{r}} \cap G_{e_{s}}$. If $r \neq 1$, then $G_{e_{r}} \cap G_{e_{s}}$ has finite index in $G_{e_{s}}$, which is a contradiction. Thus $r=1$, and similarly $s=m$.

Hence $\bigcap_{i=1}^{m-1} G_{e_{i}}$ has finite index in $G_{e_{1}}$, which implies that there is a $\kappa \in \mathbb{N}$ such that $G_{e_{1}}^{\kappa}$ is a subgroup of $\bigcap_{i=2}^{m-1} G_{e_{i}}=H$. Also $\bigcap_{i=2}^{m} G_{e_{i}}$ has finite index in $G_{e_{m}}$, which implies that there is a $\lambda \in \mathbb{N}$ such that $G_{e_{m}}^{\lambda}$ is a subgroup of $\bigcap_{i=2}^{m-1} G_{e_{i}}=H$.

On the other hand, we assumed that $\bigcap_{i=1}^{m} G_{e_{i}}$ has infinite index in every $G_{e_{i}}$ and so $\bigcap_{i=1}^{m} G_{e_{i}}$ has infinite index in both $G_{e_{1}}$ and $G_{e_{m}}$. Hence there is an element $x \in G_{e_{1}}$ of infinite order such that $\langle x\rangle \cap G_{e_{m}}=\{1\}$ and an element $y \in G_{e_{m}}$ of infinite order such that $\langle y\rangle \cap G_{e_{1}}=\{1\}$. Hence $\langle x\rangle \cap\langle y\rangle=\{1\}$. Since $G_{e_{1}}^{\kappa}$ and $G_{e_{m}}^{\lambda}$ are subgroups of $H$, we have that $\left\langle x^{\kappa}\right\rangle=\left\langle\alpha_{1}\right\rangle \subset H$ and $\left\langle y^{\lambda}\right\rangle=\left\langle\alpha_{2}\right\rangle \subset H$ with $\left\langle\alpha_{1}\right\rangle \cap\left\langle\alpha_{2}\right\rangle=\{1\}$.

Let $L$ denote the subgroup of $G$ that is generated by $H, \beta_{1} H \beta_{1}^{-1}$ and $\beta_{2} H \beta_{2}^{-1}$ where $\beta_{1} \in G_{v_{1}}$ with $\beta_{1} \notin G_{e_{1}}$ and $\beta_{2} \in G_{v_{m}}$ with $\beta_{2} \notin G_{e_{m}}$. Then, due to the choice of $\beta_{1}$ and $\beta_{2}, L$ is the fundamental group of a graph of groups with graph a tree with three vertices corresponding to the subtrees of $\Gamma, \beta_{1}\left(e_{2} \ldots e_{m-1}\right)$, $e_{2} \ldots e_{m-1}$ and $\beta_{2}\left(e_{2} \ldots e_{m-1}\right)$. The vertices that correspond to $\beta_{1}\left(e_{2} \ldots e_{m-1}\right)$ and $\beta_{2}\left(e_{2} \ldots e_{m-1}\right)$ are extremal and so $L$ is of the form

$$
L=\beta_{1} H \beta_{1}^{-1} \underset{A}{*} H \underset{B}{*} \beta_{2} H \beta_{2}^{-1} .
$$

The subgroup $A$ is the intersection of $\beta_{1} H \beta_{1}^{-1}$ and $H$. Since $H$ is the stabiliser of the subtree $e_{2} \ldots e_{m-1}, H$ fixes each of $e_{2}, \ldots, e_{m-1}$ and so $\beta_{1} H \beta_{1}^{-1}$ fixes each of $\beta_{1} e_{2}, \ldots, \beta_{1} e_{m-1}$. Hence $A$ fixes each edge in the union. Notice that the two subtrees $e_{1} \ldots e_{m-1}$ and $\beta_{1}\left(e_{1} \ldots e_{m-1}\right)$ intersect in $v_{1}$. This implies that the minimal subtree of $\Gamma$ that contains $e_{2}, \ldots, e_{m-1}$ and $\beta_{1} e_{2}, \ldots, \beta_{1} e_{m-1}$ contains $e_{1}$ and $\beta_{1} e_{1}$. Since $A$ must fix this minimal subtree, it follows that $A$ fixes $e_{1}$ and $\beta_{1} e_{1}$. 
So $A=\beta_{1} G_{e_{1}} \beta_{1}^{-1} \cap G_{e_{1}} \cap H \cap \beta_{1} H \beta_{1}^{-1}=G_{e_{1}} \cap H \cap \beta_{1} H \beta_{1}^{-1}=G_{e_{1}} \cap H$ since $G_{v_{1}}$ is abelian. Similarly, $B=G_{e_{m}} \cap H$. Then $A \cap B=H \cap G_{e_{1}} \cap G_{e_{m}}$ and since $H \cap G_{e_{1}} \cap G_{e_{m}}$ has infinite index in both $G_{e_{1}}$ and $G_{e_{m}}$, we have that $A \cap B$ has infinite index in both $A$ and $B$. But Lemma 4 implies that $L$ is not subgroup separable, a contradiction to the assumption that $G$ is subgroup separable.

$2 \Rightarrow 1$. Let $Y=T$. Then the group $N=\bigcap_{e \in E T} G_{e}$ has finite index in $G_{e}$ for at least one $e \in E T$. But $N$ is normal in $G$ because it is normal in each vertex group. So the group $G / N$ has at least one edge with finite edge group.

Let $e_{1}, \ldots, e_{m}$ be the geometric edges of $Y$ such that $G_{e_{i}} / N$ is finite for every $i=1, \ldots, m$. Then, the graph $Y \backslash\left\{e_{1}, \ldots, e_{m}\right\}$ is disconnected, consisting of connected components, some of them possibly single vertices. Notice that because of the hypothesis, for every connected component, the intersection of the edge groups that constitute the graph has finite index in some edge group, for a geometric edge in the graph. This group is again a normal subgroup of the fundamental group of the subgraph since it is normal in each vertex group of the subgraph. So we can again divide by this intersection of edge groups and repeat the above argument for every connected subgraph. The above procedure will terminate with a disconnected graph consisting of either single vertices or graphs with two edges that satisfy condition 2 of Lemma 4 Hence, all fundamental groups of the graphs of groups whose graphs are the components of the final graph are subgroup separable. Now, working backwards, we can reconstruct the group $\pi_{1}(\mathcal{G}, Y)$ and at each step use Lemma 3 along with the result of Allenby and Gregorac in 2 (that the free product of two subgroup separable groups with finite amalgamation is subgroup separable) to show that $\pi_{1}(\mathcal{G}, Y)$ is subgroup separable.

$1 \Rightarrow 3$. The result is immediate from Lemma 1.

$3 \Rightarrow 2$. Assume that $G$ does not contain a subgroup isomorphic to $L$. Let $Y$ be a subtree of $T$ such that $H=\bigcap_{e \in E Y} G_{e}$ has infinite index in every $G_{e}, e \in E Y$. Then, it was shown above that the normal closure of $H$ in $G$ contains a subgroup isomorphic to $L$, a contradiction.

Lemma 6. Let $(\mathcal{G}, X)$ be a graph of groups where $X$ consists of a single vertex $v$ such that the vertex group is a finitely generated abelian group and $n$ edge loops based at $v$ such that neither of the two injections of each edge group into the vertex group is an isomorphism. Then the following are equivalent:

(1) $G=\pi_{1}(\mathcal{G}, X)$ is subgroup separable.

(2) For every subgraph $Y$ of $X$, the group $\bigcap_{e \in E Y} G_{e}$ has a subgroup $H$ such that $H$ is normal in $G$ and $H$ has finite index in at least one $G_{e}$, for some $e \in E Y$.

(3) $G$ does not contain a subgroup isomorphic to either $L$ or $B K S$ or an ascending $H N N$-extension with finitely generated abelian base group or a nonresidually finite $H N N$-extension with finitely generated abelian base group.

Proof. $1 \Rightarrow 2$. Assume that $G$ is subgroup separable.

Let $Y$ be any subgraph of $X$. Let $C=\pi_{1}(\mathcal{G}, Y)$. Then $C$ is a $k$-fold HNNextension with base group $G_{v}$ and stable letters $t_{i}, i=1, \ldots, k$. Let $A_{i}$ and $B_{i}$ be the associated subgroups under $t_{i}$.

Take $T$ to be the $G$-tree such that $Y$ is the quotient $G \backslash T$. Choose a vertex $w$ in $T$ such that $G_{w}=G_{v}$. For each oriented edge in $Y$ there is exactly one edge in $T$ that is incident to $w$ and projects to this edge. Thus the subtree $T_{0}$ of $T$ consisting 
of all edges incident to $w$ projects down to $Y$ inducing a 2-to-1 projection on edges. Fix an $l \in\{1, \ldots, k\}$, and take $T^{\prime}$ to be the tree consisting of $T_{0}$ and the translate of $T_{0}$ by $t_{l}$. Then $T^{\prime}$ is a tree with $4 k$ vertices, since $T_{0}$ has $2 k+1$ vertices and $T_{0}$ and $t_{l} T_{0}$ meet in two points $w$ and $t_{l} w$. Let $S$ be the fundamental group of the graph of groups with graph $T^{\prime}$. Then $S$ is generated by $G_{w}, t_{i} G_{w} t_{i}^{-1}, t_{i}^{-1} G_{w} t_{i}, t_{l} t_{i} G_{w} t_{i}^{-1} t_{l}^{-1}$ and $t_{l} t_{i}^{-1} G_{w} t_{i} t_{l}^{-1}$ for every $i=1, \ldots, k$. Let us denote $D=\bigcap_{i=1}^{k}\left(A_{i} \cap B_{i}\right)$. The group

$$
M_{l}=D \cap t_{l} D t_{l}^{-1}
$$

is the intersection of all edge groups of $T^{\prime}$ and since $G$ is subgroup separable, $M_{l}$ has finite index in at least one edge group of $T^{\prime}$ by Lemma 5 . If $M_{l}$ has finite index in some $t_{l} A_{i} t_{l}^{-1}$, then it also has finite index in $A_{i}$ since $A_{i}$ and $t_{l} A_{i} t_{l}^{-1}$ have the same torsion free rank. So we can assume that $M_{l}$ has finite index in some $A_{\lambda_{l}}$ (and so in $B_{\lambda_{l}}$ ) for some $\lambda_{l} \in\{1, \ldots, k\}$. Then $t_{l}^{-1} M_{l} t_{l}$ has finite index in $t_{l}^{-1} A_{\lambda_{l}} t_{l}$. Since $A_{\lambda_{l}}$ and $t_{l}^{-1} A_{\lambda_{l}} t_{l}$ have the same torsion free rank, we have that $t_{l}^{-1} M_{l} t_{l}$ has finite index in $A_{\lambda_{l}}$. Therefore, $M_{l} \cap t_{l}^{-1} M_{l} t_{l}$ has finite index in $A_{\lambda_{l}}$ and so it also has finite index in both $M_{l}$ and $t_{l}^{-1} M_{l} t_{l}$.

Let $K$ be the subgroup of $G$ generated by $\left\langle M_{l}, t_{l}^{-1} M_{l} t_{l}\right\rangle$. Notice that both $M_{l}$ and $t_{l}^{-1} M_{l} t_{l}$ are subgroups of $D$; so both are contained in some edge group of $Y$. So, $K$ is a finitely generated abelian group since it is a subgroup of some edge group of $Y$. Moreover, the group $G_{l}$ generated by $\left\langle t_{l}, K\right\rangle$ is a subgroup of $\left\langle t_{l}, G_{w}\right\rangle$ and so has an $\mathrm{HNN}$-extension presentation

$$
G_{l}=\left\langle t_{l}, K \mid t_{l}\left(t_{l}^{-1} M_{l} t_{l}\right) t_{l}^{-1}=M_{l}\right\rangle .
$$

But by Theorem 2 in [12] there is a finite index subgroup of $M_{l}$, say $H_{l}$, such that $H_{l}$ is normalised by $t_{l}$ for every $l \in\{1, \ldots, k\}$.

Since $t_{l}^{-1} M_{l} t_{l}$ has finite index in $A_{\lambda_{l}}$ and $t_{l}^{-1} M_{l} t_{l} \subset D \subset A_{\lambda_{l}}$, we have that all $A_{\lambda_{l}}$ have the same torsion free rank as $D$. Choose $A_{\mu}$ to be one of the above $A_{\lambda_{l}}$. Then all $H_{s}$ are subgroups of finite index in $A_{\mu}$ and so we can find a finite index subgroup $H$ of $A_{\mu}$ such that $H$ is normal in $G$.

$2 \Rightarrow 1$. Use induction on the number $n$ of edges $X$ contains. If $n=1$ the result is immediate from Theorem 2 in [12].

Let $Y=X$. Then, from hypothesis, there is a finite index subgroup $H_{i}$ of some edge group $G_{e_{i}}$ such that $H_{i}$ is normal in $G=\pi_{1}(\mathcal{G}, X)$. Let $A_{i}$ and $B_{i}$ be the two isomorphic copies of $G_{e_{i}}$ embedded in $G_{v}$. Then $G / H_{i}$ is an HNN-extension of the form

$$
\left\langle t_{i}, K \mid t_{i}\left(A_{i} / H_{i}\right) t_{i}^{-1}=B_{i} / H_{i}\right\rangle
$$

where $K$ is the $(n-1)$-fold HNN-extension of $G_{v} / H_{i}$ and the groups $A_{i} / H_{i}$ and $B_{i} / H_{i}$ are finite. So, by the induction hypothesis, $K$ is subgroup separable. But from Lemma 2. $G / H_{i}^{n}$ is subgroup separable for every $n \in \mathbb{N}$. So, Lemma 3 shows that $G$ is subgroup separable.

$1 \Rightarrow 3$. Immediate.

$3 \Rightarrow 2$. Assume that $G$ does not contain any of the groups listed in the lemma. Take the normal closure of $G_{v}$ in $G$ and as before construct $M_{l}$. Since $G$ does not contain a subgroup isomorphic to $L$, by Lemma 5 , we have that $M_{l}$ has finite index in some $A_{\lambda_{l}}$. Construct again the HNN-extension

$$
G_{l}=\left\langle t_{l}, K \mid t_{l}\left(t_{l}^{-1} M_{l} t_{l}\right) t_{l}^{-1}=M_{l}\right\rangle
$$


where $K$ is the subgroup of $G$ generated by $\left\langle M_{l}, t_{l}^{-1} M_{l} t_{l}\right\rangle$. As before, both $M_{l}$ and $t_{l}^{-1} M_{l} t_{l}$ are contained in some edge group of $Y$ and so $K$ is a finitely generated abelian group. From the hypothesis that $G$ does not contain a non-residually finite HNN-extension with finitely generated abelian base group we have that $G_{l}$ is residually finite. Moreover, if $K=M_{l}$ and $t_{l}^{-1} M_{l} t_{l} \subsetneq K$ or $K=t_{l}^{-1} M_{l} t_{l}$ and $M_{l} \subsetneq K$, then $G_{l}$ is an ascending HNN-extension with finitely generated abelian base group, contained in $G$, a contradiction. Hence, either $K=M_{l}=t_{l}^{-1} M_{l} t_{l}$ or $M_{l}, t_{l}^{-1} M_{l} t_{l} \subsetneq K$. In the first case, $G_{l}$ is subgroup separable and $K$ is a normal subgroup in $G_{l}$. In the second case, we can repeat the exact argument of the proof of Theorem 2 in [12] to show that either $M_{l} \cap t_{l}^{-1} M_{l} t_{l}$ contains a subgroup, say $H_{l}$, of finite index in both $M_{l}$ and $t_{l}^{-1} M_{l} t_{l}$ such that $H_{l}$ is normal in $G_{l}$ or (if such a subgroup does not exist), to construct a subgroup of $G_{l}$ isomorphic to $B K S$. The latter is a contradiction. So, in every case, there is a normal subgroup $H_{l}$ of $G_{l}$ with finite index in $M_{l}$ for every $l \in\{1, \ldots, k\}$. Notice that if $K=M_{l}=t_{l}^{-1} M_{l} t_{l}$, then $H_{l}=K$. But $M_{l}$ has finite index in $A_{\lambda_{l}}$. So $H_{l}$ has finite index in $A_{\lambda_{l}}$. Again, all $A_{\lambda_{l}}$ have the same torsion free rank as $D$. Choose $A_{\mu}$ to be one of the $A_{\lambda_{l}}$. Then we can find a subgroup $H$ of $A_{\mu}$ such that $H$ has finite index in $A_{\mu}$ and is normal in $G$.

We are now able to prove our main theorem.

Theorem 1. Let $(\mathcal{G}, X)$ be a graph of groups such that $X$ is a finite graph and every vertex group is a finitely generated abelian group. Then the following statements are equivalent:

(1) $G=\pi_{1}(\mathcal{G}, X)$ is subgroup separable;

(2) for every connected subgraph $Y$ of $X$, there is a subgroup $H$ of the group $\pi_{1}(\mathcal{G}, Y)$ such that $H$ has finite index in at least one of the edge groups $G_{e}$, $e \in E Y$ and $H$ is normal in $\pi_{1}(\mathcal{G}, Y)$;

(3) $G$ does not contain a subgroup isomorphic to $L$ or $B K S$, nor does it contain an ascending $H N N$-extension with finitely generated abelian base group nor a non-residually finite $H N N$-extension with finitely generated abelian base group.

Proof. Let $(\mathcal{G}, X)$ be as above and delete from $X$ every edge with finite edge group. Let $\left(\mathcal{G}_{\lambda}, X_{\lambda}\right), \lambda=1, \ldots, n$, be the connected components of the new graph of groups. In view of the results in 2] and Lemma 2 $G=\pi_{1}(\mathcal{G}, X)$ is subgroup separable if and only if $\pi_{1}\left(\mathcal{G}_{\lambda}, X_{\lambda}\right)$ is subgroup separable for every $\lambda=1, \ldots, n$.

$1 \Rightarrow 2$. Let $Y$ be any subgraph of $X_{\lambda}$ and $T$ be a maximal tree of $Y$. Also let $G=\pi_{1}(\mathcal{G}, Y)$. Assume that $V Y=\left\{v_{1}, \ldots, v_{r}\right\},\left\{e_{1}, \ldots, e_{r-1}\right\} \subset E T$ such that $e_{i} \neq \overline{e_{j}}$ for every $i, j \in\{1, \ldots, r-1\}$ and $\left\{\alpha_{1}, \ldots, \alpha_{k}\right\} \subset E(Y \backslash T)$ such that $\alpha_{i} \neq \overline{\alpha_{j}}$ for every $i, j \in\{1, \ldots, k\}$. Assume, finally, that $A_{i}$ are the groups $G_{\alpha_{i}}$ and $B_{i}$ are the groups $G_{\overline{\alpha_{i}}}, i=1, \ldots, k$.

Take $\Gamma$ to be the $G$-tree such that $Y$ is the quotient $G \backslash \Gamma$ and the group associated to a vertex $v_{i}$ of $Y$ is the stabiliser of a vertex of $\Gamma$ that projects to $v_{i}$. Choose a subtree of $\Gamma$, say $\Gamma_{0}$, such that the projection of $\Gamma$ to $Y$ induces a bijection from $\Gamma_{0}$ to $T$. In this bijection, denote by $w_{i}$ the vertex of $\Gamma_{0}$ that projects to $v_{i}$ and by $f_{i}$ the edge of $\Gamma_{0}$ that projects to $e_{i}$. Finally, for every edge $\alpha_{i}$ of $Y \backslash T$ incident to $v_{j}$, choose an edge $g_{i}$ of $\Gamma \backslash \Gamma_{0}$ incident to $w_{j}$ such that $g_{i}$ projects to $\alpha_{i}$. Let $\Gamma^{\prime}$ be the subtree of $\Gamma$ with $V \Gamma^{\prime}=\left\{w_{1}, \ldots, w_{r}\right\}$ and $E \Gamma^{\prime}=\left\{f_{1}, \ldots, f_{r-1}, g_{1}, \ldots, g_{k}\right\}$. Fix an $l \in\{1, \ldots, k\}$ and take the subgroup of the normal closure of $H=\pi_{1}(\mathcal{G}, T)$, 
say $S$, that is generated by $H, t_{i} H t_{i}^{-1}, t_{i}^{-1} H t_{i}, t_{l} t_{i} H t_{i}^{-1} t_{l}^{-1}$ and $t_{l} t_{i}^{-1} H t_{i} t_{l}^{-1}$ for every $i=1, \ldots, k$. Then $S$ is the fundamental group of a graph of groups with underlying graph consisting of $\Gamma^{\prime}$ and its translate by $t_{l}$. But $S$ is a subgroup of $G$ and so is subgroup separable.

Denote $N=\bigcap_{f \in E \Gamma_{0}} G_{f}$ and $D=\bigcap_{i=1}^{k}\left(A_{i} \cap B_{i}\right)$. Then, if we apply Lemma 5 to $S$ we have that the group

$$
M_{l}=N \cap D \cap t_{l} N t_{l}^{-1} \cap t_{l} D t_{l}^{-1}
$$

has finite index in at least one of the edge groups it involves. So, $M_{l}$ has finite index in either $G_{f}$ or in $t_{l} G_{f} t_{l}^{-1}$ for some $f \in\left\{f_{1}, \ldots, f_{r-1}, g_{1}, \ldots, g_{k}\right\}$. But the torsion free rank of $t_{l} G_{f} t_{l}^{-1}$ is the same as that of $G_{f}$ and so $M_{l}$ has finite index in at least one $G_{f}, f \in\left\{f_{1}, \ldots, f_{r-1}, g_{1}, \ldots, g_{k}\right\}$.

Similarly, $t_{l}^{-1} M_{l} t_{l} \cap G_{f}$ has finite index in $G_{f}$ and so $M_{l} \cap t_{l}^{-1} M_{l} t_{l}$ has finite index in both $M_{l}$ and $t_{l}^{-1} M_{l} t_{l}$.

Since $M_{l}$ and $t_{l}^{-1} M_{l} t_{l}$ are conjugate for every $t_{l}, l=1, \ldots, k$ and commensurable, one can work as in Lemma 6] or as in the proof of Theorem 2 in [12 to obtain a finite index subgroup $H$ of some edge group $G_{f}$ such that $H$ is normal $G$.

$2 \Rightarrow 1$. We use induction on the number of geometric edges of $X_{\lambda}$. If $X_{\lambda}$ contains one edge, then $\pi_{1}\left(\mathcal{G}_{\lambda}, X_{\lambda}\right)$ is either an amalgamated free product of two abelian groups or an HNN-extension with abelian base group. In the first case, $\pi_{1}\left(\mathcal{G}_{\lambda}, X_{\lambda}\right)$ is always subgroup separable. In the second case, one can take the path in $X_{\lambda}$ that meets successively the two copies of $G_{v}$, say $A$ and $B$, associated by the stable letter. From the assumptions, $A \cap B$ has finite index in $A$ (and necessarily in $B$ ) and there is a subgroup $H$ of $A \cap B$ such that $H$ is normal in $A$ and $B$, so, by Theorem 2 in 12 is subgroup separable.

If $X_{\lambda}$ has more edges, then condition (2) states that we can find a subgroup $N$ of the group $\bigcap_{e \in E X_{\lambda}} G_{e}$ that is normal in $G$ and has finite index in some edge group $G_{\alpha}, \alpha \in E X_{\lambda}$. If $X_{\lambda} \backslash\{\alpha, \bar{\alpha}\}$ is disconnected, then $\pi_{1}\left(\mathcal{G}_{\lambda}, X_{\lambda}\right) / N$ is subgroup separable due to the inductive hypothesis and the results of [2]. If $X_{\lambda} \backslash\{\alpha, \bar{\alpha}\}$ is connected, then $\pi_{1}\left(\mathcal{G}, X_{\lambda}\right) / N$ is subgroup separable due to the inductive hypothesis and Lemma 2 Consequently, $\pi_{1}\left(\mathcal{G}_{\lambda}, X_{\lambda}\right)$ is subgroup separable from the results of Lemma 3 .

$1 \Rightarrow 3$. If $G$ is subgroup separable, then it cannot contain any of the abovementioned groups by Lemma 1

$3 \Rightarrow 2$. Let $Y$ be any subgraph of $X$. Take $T$ to be a maximal tree of $Y$ and construct $S$ as before. Using the results of Lemmas 5 and 6 one can show as before that $G$ contains a normal subgroup of $G$, say $H$, such that $H$ is of finite index in some edge group of $Y$.

Notice here that the proof of the theorem mentioned in the introduction is an immediate consequence of the above theorem.

\section{Applications}

In this section we show that the three non-subgroup separable examples of [13], [1] and [9] can be treated using our techniques. More specifically, in Corollary [1] we use our method of proof to show that $F_{2} \times F_{2}$ contains a subgroup isomorphic to $L$ in [14]. In Corollaries 2 and 3 we show that the examples presented there are special cases of our theorem. 
Corollary 1 (13]). The group $G=F_{2} \times F_{2}$ is not subgroup separable.

Proof. Take two copies of $F_{2}$ with generators $\left\langle x_{1}, x_{2}\right\rangle$ and $\left\langle y_{1}, y_{2}\right\rangle$. Then the subgroup of $G$, say $H$, generated by $H=\left\langle x_{1}, y_{1}\right\rangle$ is free abelian of rank two. The normal closure of $H$ in $G$ contains a subgroup of the form

$$
y_{2} H y_{2}^{-1} \underset{y_{2} x_{1} y_{2}^{-1}=x_{1}}{*} H \underset{y_{1}=x_{2} y_{1} x_{2}^{-1}}{*} x_{2} H x_{2}^{-1} .
$$

But the above group is isomorphic to $L$ and so it cannot be subgroup separable; hence $G$ is not subgroup separable.

Corollary 2 (1]). Let $A$ be the group with presentation

$$
A=\left\langle a, d_{1}, d_{2}, d_{3} \mid\left[d_{i}, d_{j}\right]=1, a d_{1} a^{-1}=d_{1} d_{2}^{2},\left[a, d_{2}\right]=\left[a, d_{3}\right]=1\right\rangle
$$

and let $B$ be the group with presentation $B=\langle a, c \mid[a, c]=1\rangle$. Then the group $G=A \underset{\langle a\rangle}{*} B$ is not subgroup separable.

Proof. The group $G$ can be written as an HNN-extension of the form

$$
G=\left\langle d_{1}, c, K \mid d_{1}^{-1} A_{1} d_{1}=B_{1}, c^{-1} A_{2} c=B_{2}\right\rangle
$$

where $K=\left\langle a, d_{2}, d_{3} \mid\left[a, d_{2}\right]=\left[a, d_{3}\right]=\left[d_{2}, d_{3}\right]=1\right\rangle$ and $A_{1}=\left\langle a, d_{2}, d_{3}\right\rangle, B_{1}=$ $\left\langle d_{2}^{2} a, d_{2}, d_{3}\right\rangle, A_{2}=\langle a\rangle=B_{2}$. For $G$ to be subgroup separable, Theorem 1 requires the existence of a subgroup $H$ of $K$ such that $H$ has finite index in either $A_{1}$ or $A_{2}$ and such that $H$ is fixed under the isomorphisms $\phi_{i}: A_{i} \rightarrow B_{i}, i=1,2$. One can easily see that the only candidate for $H$ is $H=\left\langle a^{k}\right\rangle, k \in \mathbb{N}$. But $H$ cannot be fixed by $\phi_{1}$ and so such an $H$ cannot exist. Hence, $G$ is not subgroup separable.

Corollary 3 (9). Let $D$ be a free abelian group with the basis $d_{1}, d_{2}, d_{3}, d_{4}, d_{5}$, $d_{6}, d_{7}, d_{8}$. Let $A=D \rtimes\langle a\rangle$, where $a^{-1} d_{1} a=d_{1} d_{2}, a^{-1} d_{2} a=d_{2}, a^{-1} d_{3} a=d_{4}$, $a^{-1} d_{4} a=d_{3}, a^{-1} d_{5} a=d_{6}, a^{-1} d_{6} a=d_{5}, a^{-1} d_{7} a=d_{7}, a^{-1} d_{8} a=d_{8}$.

Let $B$ be any group containing an element $b$ of infinite order. If $B$ contains an element $c$ such that $b c=c b$ and $c \notin\langle b\rangle$, then $A \underset{a=b}{*} B$ is not subgroup separable.

Proof. The subgroup of $G$, say $H$, generated by $\left\langle d_{1}, d_{2}, a, c d_{2}\right\rangle$ has a presentation of the form

$$
H=\left\langle d_{1}, c d_{2}, K \mid d_{1}^{-1} A_{1} d_{1}=B_{1}, c d_{2} A_{2}\left(c d_{2}\right)^{-1}=B_{2}\right\rangle
$$

where $K=\left\langle d_{2}, a \mid\left[d_{2}, a\right]=1\right\rangle=A_{1}=B_{1}$ and $A_{2}=\langle a\rangle=B_{2}$. As before, for $H$ to be subgroup separable, Theorem 1 requires the existence of a normal subgroup $M$ such that either $A_{1} / M$ or $A_{2} / M$ is finite. So, the only possible candidate would be the groups $\left\langle a^{k}\right\rangle, k \in \mathbb{N}$. But the groups $\left\langle a^{k}\right\rangle$ cannot be fixed by the isomorphism between $A_{1}$ and $B_{1}$. So, such an $M$ does not exist. Hence, $H$ is not subgroup separable and neither is $G$.

\section{ACKNOWLEDGMENTS}

The authors wish to thank the first referee for spotting a mistake in the previous version of the paper and for several amendments and the second referee for several corrections and for helping us to improve our exposition. 


\section{REFERENCES}

[1] R.B.J.T. Allenby and D. Doniz, A free product of finitely generated nilpotent groups amalgamating a cycle that is not subgroup separable, Proc. Amer. Math. Soc. 124, 1003-1005 (1996). MR 97a:20049

[2] R.B.J.T. Allenby and R. J. Gregorac, On locally extended residually finite groups, Lecture Notes in Mathematics, Vol. 319, Springer-Verlag, Berlin, 1973, pp. 9-17. MR 52:3333

[3] S. Andreadakis, E. Raptis, and D. Varsos, A characterisation of residually finite HNNextensions of finitely generated abelian groups, Arch. Math. (Basel) 50, 495-501 (1988). MR 89g:20046

[4] M. Bestvina and N. Brady, Morse theory and finiteness properties of groups, Invent. Math. 129, 445-470 (1997). MR 98i:20039

[5] A. Blass and P. M. Neumann, An application of universal algebra in group theory, Michigan Math. J. 21, 167-169 (1974). MR 51:729

[6] R. G. Burns, A. Karrass, and D. Solitar, A note on groups with separable finitely generated subgroups, Bull. Austral. Math. Soc. 36, 153-160 (1987). MR 88g:20057

[7] D. E. Cohen, Combinatorial group theory: a topological approach, London Mathematical Society Student Texts 14, 1989. MR 91d:20001

[8] R. Gitik, Graphs and separability properties of groups, J. Algebra 188, 125-143 (1997). MR 98e:20033

[9] R. Gitik and E. Rips, A necessary condition for $A \underset{a=b}{*} B$ to be LERF, Israel J. Math. 73, 123-125 (1991). MR 92g:20046

[10] M. Hall, Jr., Coset representations in free groups, Trans. Amer. Math. Soc. 67, 421-432 (1949). MR 11:322e

[11] D. D. Long and G. A. Niblo, Subgroup separability and 3-manifold groups, Math. Z. 207, 209-215 (1991). MR 92g:20047

[12] V. Metaftsis and E. Raptis, Subgroup separability of HNN-extensions with abelian base group, J. Algebra 245, 42-49 (2001). MR 2002j:20050

[13] K. A. Mihailova, The occurrence problem for direct products of groups, Mat. Sbornik 70, 241-251 (1966). MR 33:2707

[14] G. Niblo and D. Wise, Subgroup separability, knot groups and graph manifolds, Proc. Amer. Math. Soc. 129, 685-693 (2001). MR 2001f:20075

[15] J.-P. Serre, Trees, Springer-Verlag, Berlin and New York, 1980. MR 82c:20083

[16] P. Scott, Subgroups of surface groups are almost geometric, J. London Math. Soc. 17, 555-565 (1978). MR 58:12996

[17] P. Scott, Correction to "Subgroups of surface groups are almost geometric", J. London Math. Soc. 32, 217-220 (1985). MR 87k:57003

[18] P. B. Shalen, Three-manifolds and Baumslag-Solitar groups, Geometric Topology and Geometric Group Theory (Milwaukee, WI, 1997). Topology Appl. 110, 113-118 (2001). MR 2001j:57002

[19] D. T. Wise, Subgroup separability of graphs of free groups with cyclic edge groups, Quart. J. Math. 51, 107-129 (2000). MR 2001e:20026

Department of Mathematics, University of the Aegean, Karlovassi, 83200 Samos, Greece

E-mail address: vmet@aegean.gr

Department of Mathematics, University of Athens, Panepistimiopolis, 15784 Athens, GREeCE

E-mail address: eraptis@math.uoa.gr 